\title{
COMPARISON OF ASTROMETRICAL ACCURACY OF BIG SCHMIDT TELESCOPES
}

\author{
E. SCHILBACH ${ }^{1}$, S. HIRTE ${ }^{1}$, AND J.-L. HEUDIER ${ }^{2}$ \\ 1 Zentralinstitut für Astrophysik \\ Rosa-Luxemburg-Strasse 17A \\ 1591 Potsdam \\ GERMANY, Dem. Rep. \\ ${ }^{2}$ Observatoire de la Côte d'Azur, \\ Caussols, France
}

\begin{abstract}
The measurements of AGK3 stars carried out with the automated measuring machine MAMA in Paris were used for the estimation of the accuracy of stellar positions on Tautenburg and CERGA plates. The results show good coincidence of stellar positions derived with both Schmidt telescopes. The achieved accuracy is high enough to use combined observations for improving present positions in the catalog AGK3.
\end{abstract}

\section{Plate Material}

The use of big Schmidt telescopes and automated measuring machines enables us to derive positions of great numbers of objects. The accuracy of measurements depends on the quality of the images and is, generally, best for faint $\left(15^{\mathrm{m}}-17^{\mathrm{m}}\right)$ stars. However, to get equatorial coordinates of interesting objects we have to measure also bright $\left(7^{\mathrm{m}}-12^{\mathrm{m}}\right)$ reference stars.

In order to estimate the accuracy of stellar positions to be achieved in this situation we used plates taken with the Schmidt telescopes at Tautenburg (134/203/401) and at Caussols, CERGA (90/152/ 316). In the description of the telescopes, the first number denotes the diameter of the correction plate, the second denotes the diameter of the mirror, and the third denotes the focal length.

Three plates centered at $\eta$ Tau (Pleiades) were taken at nearly the same time and in the same color system, but with exposure times of 5 minutes for CERGA and 20 minutes for Tautenburg plates. The CERGA plates $\left(30 \times 30 \mathrm{~cm}^{2}\right)$ and the Tautenburg plates $\left(24 \times 24 \mathrm{~cm}^{2}\right)$ cover sky areas of $5.2 \times 5.2$ and $3: 2 \times 30^{\circ} 2$, respectively. In a field of $3^{\circ} \times 3^{\circ} 133$ AGK3 stars fainter than the 7 th magnitude were measured with the automated measuring machine maMa in Paris. The AGK3 coordinates were reduced to the epoch of observation by applying AGK3 proper motions and thereafter transformed into a plate-centered zenith-azimuth system.

\section{Results}

The form of the reduction polynomials was established by means of the method of stepwise regression (Hirte et al. 1989) for each plate separately. The starting model was fixed by the complete third-order polynomial in powers of coordinates. In this case the stepwise regression allows us to 
find the "best" model with only significant terms among all polynomials up to the third order.

In order to estimate the accuracy of positions, the differences between the catalog coordinates of 133 AGK3 stars and their measured coordinates transformed into the system of the catalog were analysed. Under the assumption that all systematic effects are taken into account by the reduction model, these differences DX and DY may be considered as random values.

First, we compared the differences for each star measured on the Tautenburg and CERGA plates separately. For three Tautenburg plates, the errors of a stellar position range from 0."04 to 0". 10 with the mean values of 0."05 $(1.0 \mu \mathrm{m})$ in X (RA) and 0.07 $(1.5 \mu \mathrm{m})$ in Y (declination). For the CERGA plates, a positional accuracy of 0.05 to 0."13 with the mean values of $0.09(1.4 \mu \mathrm{m})$ in X and 0.08 $(1.2 \mu \mathrm{m})$ in $\mathrm{Y}$ was achieved.

These results were used to estimate the error $R$ which may arise if positions are determined on plates taken with different telescopes:

$$
m_{\mathrm{TC}}^{2}=m_{\mathrm{T}}^{2}+m_{C}^{2}+R^{2}
$$

where $m_{\mathrm{T}}$ and $m_{\mathrm{c}}$ are mean errors of a stellar position on a Tautenburg and on a CERGA plate, respectively, and $m_{\mathrm{TC}}$ is a mean error of the corresponding difference of coordinates. Considering all nine combinations of plates we obtained $m_{\mathrm{TC}}$ varying from 0.10 to 0 ". 16 with the mean values of 0 ". 12 and 0.13 in $X$ and $Y$, respectively. The error $R$ ranges from $0: 0$ to 0.11 . The mean value of $R$ was found to be 0.07 in $\mathrm{X}$ and 0.05 in $\mathrm{Y}$.

Finally, the mean error of a position in the AGK3 was estimated as

$$
m_{\mathrm{K}}^{2}=0.5\left(m_{\mathrm{KT}}^{2}+m_{\mathrm{KC}}^{2}-m_{\mathrm{TC}}^{2}\right)
$$

where $m_{\mathrm{K}}$ is a mean error of a catalog position and $m_{\mathrm{KC}}$ and $m_{\mathrm{KT}}$ are mean errors of the differences between catalog and plate coordinates( $\mathrm{C}$ - CERGA, T - Tautenburg). For nine plate pairs we obtained an accuracy of a catalog position in the Pleiades region between 0.47 and 0.48 with the mean value of 0.47 in $\mathrm{X}$ and between 0.50 and 0.52 with the mean value of 0.50 in $\mathrm{Y}$.

These results coincide well with the value of 0.45 found by Bastian (1989) as the average of the mean errors of position at epoch 1990.0 for the catalog AGK3. The achieved accuracy is high enough to use combined observations with the Tautenburg and CERGA telescopes for improving the proper motions in the catalog AGK3. The fact that nearly the same measuring accuracy was achieved on the plates independently from exposure time exhibits the good quality of the measuring machine MAMA. An analogous investigation based on these plates and on the catalog by Eichhorn et al. (1970) as reference catalog is now in preparation. Preliminary tests show a good agreement between catalog and measured coordinates. We intend to use the measurements of the plates to derive the presently missing proper motions for ca. 400 stars in Eichhorn's catalog.

\section{References}

Bastian, U.: 1989, "PPM: a tool for astronomers", in Inertial Coordinate System on the Sky, IAU Symposium No. 141 (this volume).

Eichhorn, H., Googe, W.D., Lukac, C.F., Murphy, J.K.: 1970, “Accurate positions of 502 stars in the region of the Pleiades", Mem. Roy Astron. Society 73, 125.

Hirte, S., Dick, W.R., Schilbach, E., Scholz, R.-D.: 1989, In: Errors, Uncertainties and Bias in Astronomy, C. Jaschek and F. Murtagh (Eds.), Cambridge University Press, in press. 